\title{
BESIII results on time-like baryon form factors
}

\author{
Alaa Dbeyssi ${ }^{1, \star}$ (on behalf of BESIII collaboration) \\ ${ }^{1}$ Helmholtz-Institut Mainz, Germany
}

\begin{abstract}
This contribution reports on the recent measurements of baryon electromagnetic form factors at the BESIII experiment in Beijing. The Beijing $e^{+} e^{-}$collider BEPCII is a double-ring symmetric collider running at $\sqrt{s}$ between 2.0 and $4.6 \mathrm{GeV}$. Baryon electromagnetic form factors can be measured at BESIII in direct $e^{+} e^{-}$-annihilation and in initial state radiation processes. Based on the data collected by the BESIII detector at 12 center of mass energies between 2.23 and $3.67 \mathrm{GeV}$, the measured $e^{+} e^{-} \rightarrow \bar{p} p$ cross section and the time-like proton form factor are presented. Preliminary results from the analysis of the initial state radiation process $e^{+} e^{-} \rightarrow \bar{p} p \gamma$ using a data set of $7.408 \mathrm{fb}^{-1}$ collected at center of mass energies between 3.773 and $4.6 \mathrm{GeV}$, are also shown. The cross sections for $e^{+} e^{-} \rightarrow \bar{\Lambda}_{c}^{-} \Lambda_{c}^{+}$and $e^{+} e^{-} \rightarrow \bar{\Lambda} \Lambda$ are measured near threshold with unprecedented precision. Preliminary results on the first measurement of the $\Lambda_{c}$ form factor ratio $\left|G_{E}\right| /\left|G_{M}\right|$ are also given. Ongoing analysis based on the high luminosity energy scan from 2015 and from radiative return at different $\sqrt{s}$ are also described.
\end{abstract}

\section{Introduction}

Electromagnetic form factors are fundamental quantities, which describe the distribution of the electric charges and magnetization within the hadrons. Baryons, spin 1/2 particles, are characterised by the electric $G_{E}\left(q^{2}\right)$ and magnetic $G_{M}\left(q^{2}\right)$ form factors. The electromagnetic form factors of baryons can be measured in time-like region (momentum transfer $q^{2}<0$ ) through the annihilation reactions $e^{+} e^{-} \leftrightarrow \bar{B} B$ using the energy scan technique, in which the center of mass energy of the collider $(\sqrt{s})$ is varied systematically, and at each energy point a measurement of the associated cross section can be carried out. It is assumed that the interaction occurs through the exchange of one photon, which carries the momentum transfer squared $q^{2}$. The initial state radiation (ISR) technique is also an effective tool to measure hadronic cross section at high luminosity $e^{+} e^{-}$-storage rings, such as the Beijing Electron-Positron Collider II (BEPCII). Due to the fact that annihilation of $e^{+} e^{-}$is mostly accompanied by emission of one or several photons from the initial state, the high luminosity collected at the $e^{+} e^{-}$collider experiments, allows for a complementary approach to the energy scan technique in hadron cross section measurements: events with ISR photons lead to a reduction of the invariant mass $\left(q^{2}\right)$ of the proton-antiproton system, and hence allow for a measurement of the baryonic form factors over a wide range of $q^{2}$ below $\sqrt{s}$.

\footnotetext{
^e-mail: adbeyssi@uni-mainz.de
} 


\section{BESIII detector and data sets}

The BESIII detector [1] is a general purpose spectrometer located at BEPCII. BEPCII is a double ring $e^{+} e^{-}$collider running at center of mass energies $(\sqrt{s})$ between 2.0 and $4.6 \mathrm{GeV}$ and reached a peak luminosity of $1.0 \times 10^{33} \mathrm{~cm}^{-2} \mathrm{~s}^{-1}$ at $\sqrt{s}=3770 \mathrm{MeV}$. The cylindrical BESIII detector has an effective geometrical acceptance of $93 \%$ of $4 \pi$. It contains a small cell, helium-based $\left(60 \% \mathrm{He}, 40 \% \mathrm{C}_{3} \mathrm{H}_{8}\right)$ main drift chamber (MDC) which provides momentum measurements of charged particles with a resolution of $0.5 \%$ at $1 \mathrm{GeV} / \mathrm{c}$ in a 1 Tesla magnetic field. The energy loss $(\mathrm{dE} / \mathrm{dx})$ measurement provided by the MDC has a resolution better than $6 \%$. A time-of-flight system (TOF) consisting of 5-cm-thick plastic scintillators with a time resolution of $80 \mathrm{ps}$ in the barrel and $70 \mathrm{ps}$ in the end caps. An electromagnetic calorimeter (EMC) consisting of $6240 \mathrm{CsI}$ (Tl) in a cylindrical structure and two end caps is used to measure the energies of photons and electrons. The energy resolution of EMC (for $1 \mathrm{GeV}$ electrons and photons) is $2.5 \%$ in the barrel and $5.0 \%$ in the end caps. The position resolution of EMC is $6 \mathrm{~mm}$ in the barrel and $9 \mathrm{~mm}$ in the end caps for $1 \mathrm{GeV}$ electrons and photons. A muon system (MUC) consisting of $1000 \mathrm{~m}^{2}$ of Resistive Plate Chambers (RPC) is used to identify muons and provides a spatial resolution better than $2 \mathrm{~cm}$.

BESIII provided the world's largest samples of $e^{+} e^{-}$collisions in the $\tau$-charm region. Data samples have been accumulated at different charmonium and X, Y, Z states, and high luminosity scan experiments have been performed in the region between 2.0 and $4.6 \mathrm{GeV}$. The data collected at BESIII allows the measurement of baryon form factors using the direct baryon pair productions and the initial state radiation processes.

\section{Measurements of proton form factors at BESIII}

\subsection{Measurement of the $e^{+} e^{-} \rightarrow \bar{p} p$ using the scan technique}

The cross section of $e^{+} e^{-} \rightarrow \bar{p} p$ is measured by BESIII at 12 center of mass energies between 2.2 and $3.7 \mathrm{GeV}$ using $157 \mathrm{pb}^{-1}$ data samples [2]. The event selection criteria, the estimation of the background contamination and the evaluation of the signal efficiencies are obtained based on the Monte Carlo simulations for the signal and the different background processes. The Born cross section of $e^{+} e^{-} \rightarrow \bar{p} p$ is calculated by:

$$
\sigma_{\text {Born }}=\frac{N_{o b s}-N_{b k g}}{L \epsilon(1+\delta)},
$$

where $N_{o b s}$ is the number of selected events, $N_{b k g}$ is the estimated number of background events, $L$ is the integrated luminosity determined at each center of mass energy, $\epsilon$ is the detection efficiency of the signal and $(1+\delta)$ is the radiative correction factor calculated using the CONEXC event generator [3]. An effective electromagnetic form factor $\left(\left|G_{e f f}\right|\right)$ of the proton can be deduced from the measured Born cross section using:

$$
\begin{aligned}
\sigma\left(q^{2}\right) & =\frac{2 \pi \alpha^{2} \beta C}{3 q^{2} \tau}\left(2 \tau\left|G_{M}\right|^{2}+\left|G_{E}\right|^{2}\right), G_{e f f} \mid=\sqrt{\frac{2 \tau\left|G_{M}\right|^{2}+\left|G_{E}\right|^{2}}{2 \tau+1}} \\
\beta & =\sqrt{1-\frac{1}{\tau}}, \tau=\frac{q^{2}}{4 M^{2}}, C=\frac{y}{(1-\exp (-y))}, y=\frac{\alpha \pi}{\beta},
\end{aligned}
$$

where $\alpha$ is the electromagnetic coupling constant and $C$ is the Coulomb factor for the case of pointlike fermions [4]. The results on the Born cross section are shown in Fig. 1(a) together with the previous experimental measurements. Below $\sqrt{s}=3.08 \mathrm{GeV}$, BESIII improves the precision on the Born cross section by $30 \%$ compared to the most precise data measured by BaBar $[5,6]$. 


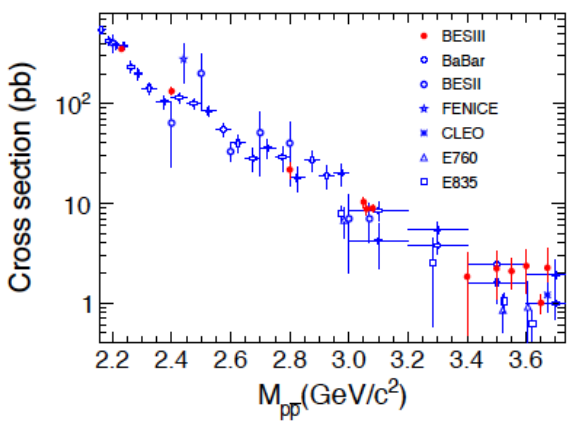

(a)

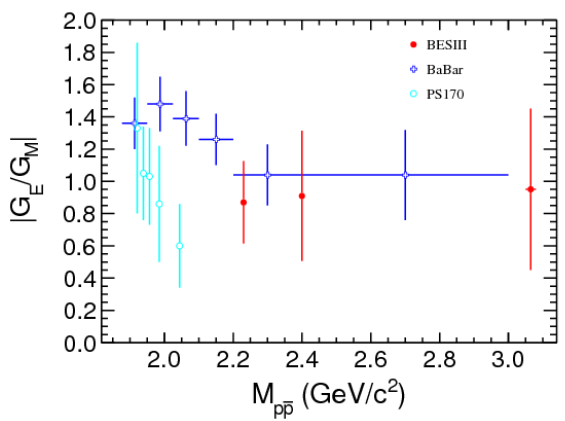

(b)

Figure 1. Comparison of the (a) Born cross section of $e^{+} e^{-} \rightarrow \bar{p} p$ and (b) the proton form factor ratio between BESIII (red points) and previous measurements.

The ratio of the proton form factors is extracted from the fit to the distribution of the proton polar angle using the large data samples collected at $\sqrt{s}=2232.4,2400.0$ and a combined sample at $\sqrt{s}=3050.0,3060.0$ and $3080.0 \mathrm{MeV}$. The measured ratio of the proton form factors (Fig. 1(b)) is close to unity and it is consistent with the BABAR results in the same $q^{2}$ region.

\subsection{Measurement of the $e^{+} e^{-} \rightarrow \bar{p} p$ using the ISR technique}

Based on $7.408 \mathrm{fb}^{-1}$ data samples collected with the BESIII detector at center of mass energies between 3.773 and $4.600 \mathrm{GeV}$, the proton form factors have been measured using the initial state radiation technique. In this work, the two charged tracks (proton and antiproton) are reconstructed with the MDC, and the ISR photon is assigned to the highest energy photon detected by the EMC. Only the events where the ISR photons are emitted within the acceptance of the EMC are selected (tagged ISR analysis). Signal and background processes have been simulated with the BESIII Offline Software System (BOSS) [7] which includes full detector simulation and event reconstruction. The ratio of the proton form factors has been extracted in six intervals of $\bar{p} p$-invariant mass from the threshold up to $3.0 \mathrm{GeV} / \mathrm{c}^{2}$. The Born cross section of $e^{+} e^{-} \rightarrow \bar{p} p$ and the proton effective form factor have been also measured in 31 intervals of $\bar{p} p$-invariant mass below $3.0 \mathrm{GeV} / \mathrm{c}^{2}$.

Figs. 2(a) and 2(b) show the preliminary results for the Born cross section of $e^{+} e^{-} \rightarrow \bar{p} p$ and the proton form factor ratio, respectively. The results are consistent with the previous experiments. The total uncertainty, dominated by the statistical one, is between $20 \%$ and $35 \%$ for the ratio and between $10 \%$ and $64 \%$ for the Born cross section. The Coulomb factor (Eq. 2) predicts a non vanishing cross section at threshold followed by a sharp increase in the cross section of $e^{+} e^{-} \rightarrow \bar{p} p$. The experimental data (Fig. 2(a)) show a flat distribution near threshold which could be explained by a sharp decrease of the proton effective form factor near threshold.

The $e^{+} e^{-} \rightarrow p \bar{p} \gamma$ events in which the ISR photons cannot be detected by the EMC have been also analysed (untagged ISR analysis). The measurement of these events benefits from the fact that the number of statistics is increased by a factor $\sim 3$ compared to the tagged analysis. However, the difficulty of this measurement relays on the signal/background separation using only the information on two detected particles, proton and anti-proton. Based on the Monte Carlo simulations, using PHOKHARA 9.1 event generator [8], the Born cross section of $e^{+} e^{-} \rightarrow p \bar{p}$ can be measured in the region between 2 and $\sim 4 \mathrm{GeV} / c^{2}$, with a statistical accuracy between $5 \%$ and $35 \%$. 


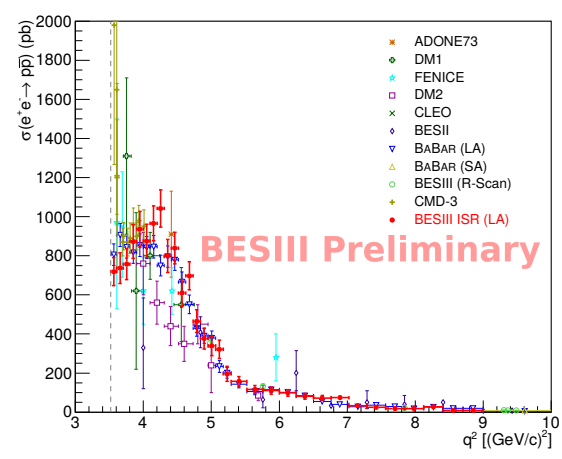

(a)

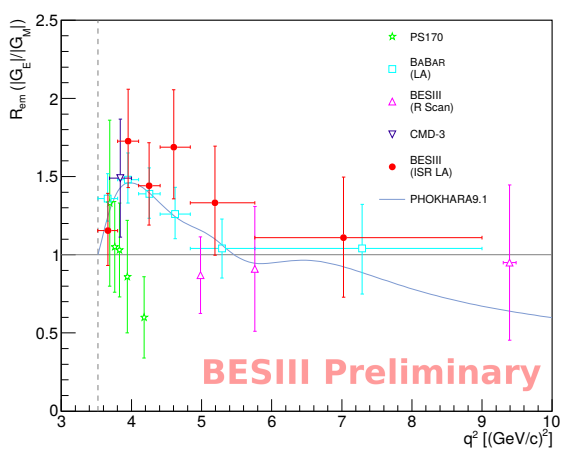

(b)

Figure 2. (a) BESIII preliminary results on the Born cross section of $e^{+} e^{-} \rightarrow \bar{p} p$ (BESIII ISR (LA), red points) and (b) on the the ratio of the proton form factors from the tagged ISR analysis .

\section{Hyperon form factors at BESIII}

The experimental measurements of the time-like proton form factors show a strong energy dependance and some unexpected features near the threshold region [9-11]. A non vanishing cross section of the $e^{+} e^{-} \rightarrow p \bar{p}$ process followed by a plateau is observed near threshold (Fig. 2(a)). This could be a general feature for baryons in the time-like region. In this context, a precise and complete measurement of other baryon form factors like for neutron, charged and neutral hyperons is of great importance. Preliminary results on the $e^{+} e^{-} \rightarrow \bar{\Lambda}_{c}^{-} \Lambda_{c}^{+}$and $e^{+} e^{-} \rightarrow \bar{\Lambda} \Lambda$ cross sections have been recently released by the BESIII Collaboration. The Born cross section for the channel $e^{+} e^{-} \rightarrow \bar{\Lambda}_{c}^{-} \Lambda_{c}^{+}$is measured at BESIII using the data samples collected at 4 center of mass energies $\sqrt{s}=4.5745,4.5800,4.5900$ and $4.5995 \mathrm{GeV}$. These are the first measurements of the $e^{+} e^{-} \rightarrow \bar{\Lambda}_{c}^{-} \Lambda_{c}^{+}$cross section in direct baryon pair production (scan mode). The results are shown in Fig. 3 together with the previous ISR measurements from the Belle Collaboration [12]. The achieved statistical uncertainty on the cross section measurement at $4.5995 \mathrm{GeV}$ is only $\sim 1.3 \%$. The Born cross section of $e^{+} e^{-} \rightarrow \bar{\Lambda}_{c}^{-} \Lambda_{c}^{+}$is suddenly different from zero at threshold and follows a flat behaviour, like the case of $e^{+} e^{-} \rightarrow p \bar{p}$ process, in the near threshold region.

The high luminosity values $47.67 \mathrm{pb}^{-1}$ and $566.9 \mathrm{pb}^{-1}$ collected at $\sqrt{s}=4.5745$ and $4.5995 \mathrm{GeV}$ respectively, allow for a first time measurement of the form factor ratio $\left(\left|G_{E}\right| /\left|G_{M}\right|\right)$ for the charmed hyperon $\Lambda_{c}$. The measured values are $\left|G_{E}\right| /\left|G_{M}\right|=1.14 \pm 0.14 \pm 0.07$ and $\left|G_{E}\right| /\left|G_{M}\right|=1.23 \pm 0.05 \pm 0.03$ at $\sqrt{s}=4.5745$ and $4.5995 \mathrm{GeV}$ respectively (preliminary results).

Data samples of $40.5 \mathrm{pb}^{-1}$ collected at $\sqrt{s}=2.2324,2.400,2.800$ and $3.080 \mathrm{GeV}$ have been analyzed for the measurement of the the $e^{+} e^{-} \rightarrow \bar{\Lambda} \Lambda$ cross section. The lowest energy point, 2.2324 $\mathrm{GeV}$, is only 1.0 MeV above the threshold of the $\bar{\Lambda} \Lambda$ production. At this energy, the $e^{+} e^{-} \rightarrow \bar{\Lambda} \Lambda$ process is analyzed from two decay modes, $\bar{p} p \pi^{+} \pi^{-}$and the inclusive $\bar{n} \pi^{0}+X$. The measurement of the cross section of $e^{+} e^{-} \rightarrow \bar{\Lambda} \Lambda$ process from the two decay modes gives consistent results. At $\sqrt{s}=2.400,2.800$ and $3.080 \mathrm{GeV}$, the cross section is measured using only the charged decay mode $\left(\bar{p} p \pi^{+} \pi^{-}\right)$. The preliminary results on the Born cross section and the hyperon effective form factor are shown in Fig. 4. At $\sqrt{s}=2.2324 \mathrm{GeV}$, the average cross section is $318 \pm 60 \mathrm{pb}$, which is significantly larger than the expected value assuming no Coulomb interaction between the produced neutral hyperons. At the higher energy points, the results are consistent with the previous data. 


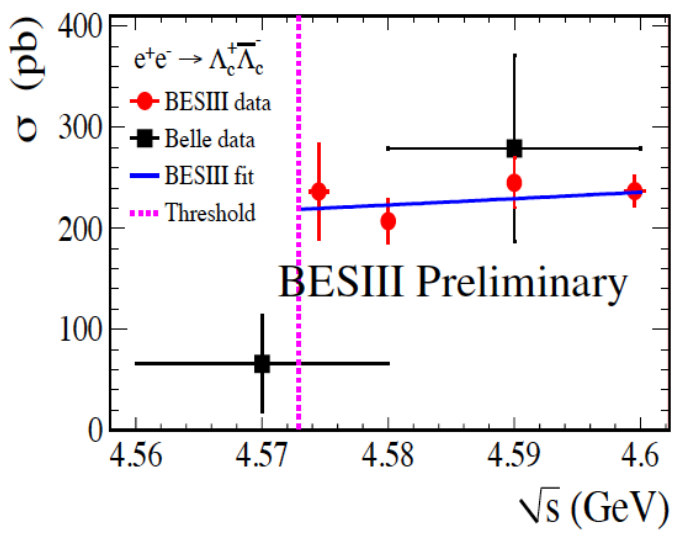

Figure 3. BESIII preliminary results (red points) on the Born cross section of $e^{+} e^{-} \rightarrow \bar{\Lambda}_{c}^{-} \Lambda_{c}^{+}$.
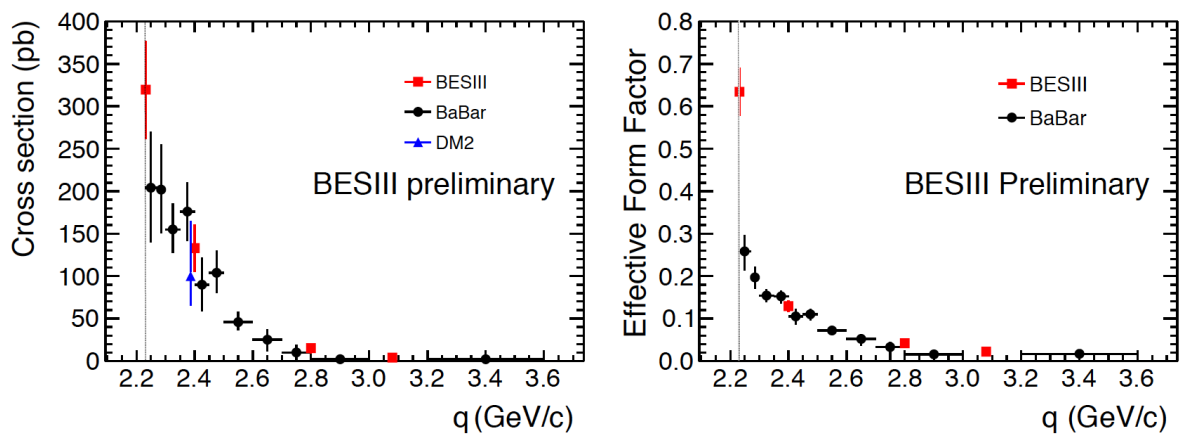

Figure 4. (a) BESIII preliminary results (red points) on the Born cross section of $e^{+} e^{-} \rightarrow \bar{\Lambda} \Lambda$ and (b) on the $\Lambda$ effective form factor.

\section{Prospects}

BESIII is an excellent laboratory for the measurement of baryon time-like form factors. Both ISR and scan methods can be performed, and the kinematical threshold for different baryon pair productions is covered by the energy range of BEPCII. In 2015, BESIII performed high luminosity scan in 22 energy points between 2.0 and $3.08 \mathrm{GeV}$. Based on these data samples, the proton electromagnetic form factors $\left|G_{E}\right|$ and $\left|G_{M}\right|$ can be extracted separately. The expected precision on the ratio of the proton form factors is at the level of $10 \%$. In addition to the proton, BESIII can provide the most precise measurements of the neutron form factors with the $e^{+} e^{-} \rightarrow \bar{n} n$ and $e^{+} e^{-} \rightarrow \bar{n} n \gamma$ processes. The determination of $\Lambda$ form factors, $\left|G_{E}\right|,\left|G_{M}\right|$ and the relative phase between them is also possible. The polarization of $\Lambda$ in $e^{+} e^{-} \rightarrow \bar{\Lambda} \Lambda$, which contains information on the relative phase between $\left|G_{E}\right|$ and $\left|G_{M}\right|$, can be experimentally accessed thanks to the self-analyzing weak decay of $\Lambda$, i.e $\Lambda \rightarrow p \pi^{-}$. The measurement of the hyperons form factors at BESIII can be extended to other channels, i.e. $e^{+} e^{-} \rightarrow \bar{\Sigma} \Sigma, \Lambda \bar{\Sigma}^{0}, \bar{\Omega} \Omega \ldots$ 


\section{References}

[1] M. Abilikim et al. (BESIII Collaboration), Nucl. Instru. Meth. A 614, 345 (2010).

[2] M. Abilikim et al. (BESIII Collaboration), Phy. Rev D 91, 112004 (2015).

[3] R. G. Ping, Chin. Phys. C 38, 083001 (2014).

[4] C. Tzara, Nucl. Phys. B 18 (1970) 216-252.

[5] J. P. Lees et al. (BABAR Collaboration), Phys. Rev. D 87, 092005 (2013).

[6] J. P. Lees et al. (BABAR Collaboration), Phys. Rev. D 88, 072009 (2013).

[7] Z. Y. Deng, et al., HEP\&NP. 30, 371 (2006).

[8] H. Czyt, M. Gunia and J. H. Kuehn, JHEP 1308, 110 (2013).

[9] R. Baldini, S. Pacetti, A. Zallo and A. Zichichi, Eur. Phys. J. A 39, 315 (2009);

[10] A. Bianconi and E. Tomasi-Gustafsson. Phys. Rev. C 93, 035201 (2016).

[11] J. Haidenbauer and U. G. Meissner, Phys. Lett. B 761, 456 (2016).

[12] G. Pakhlova et al. (Belle Collaboration), Phys. Rev. Lett. 101, 172001 (2008). 